\title{
EFICIÊNCIA AGRONÔMICA DA CULTURA DA SOJA (Glycine max (L.) Merril) SUBMETIDA A COINOCULAÇÃO
}

\author{
Agronomic efficiency of soybean culture (Glycine max (L.) Merril) submitted to coinoculation.
}

Fernando Schneider ${ }^{1}$; Luis Carlos Panizzon²; André Sordi ${ }^{3 *}$; Cristiano Reschke Lajús ${ }^{4}$; Alceu Cericato ${ }^{5}$; Claudia Klein ${ }^{6}$.

\author{
${ }^{1}$ Engenheiro Agrônomo. E-mail: fernando sch@outlook.com. \\ 2 Engenheiro Agrônomo. E-mail: luiscarlos.panizzon@hotmail.com. . \\ 3 Professor do Curso de Agronomia, Universidade do Oeste de Santa Catarina- UNOESC. - São José do Cedro - SC; Brasil. E- \\ mail: andresordi@yahoo.com.br \\ * Autor para correspondência \\ ${ }^{4}$ Professor do Curso de Agronomia, Universidade do Oeste de Santa Catarina-UNOESC - São José do Cedro - SC; Brasil. E- \\ mail.crlajus@hotmail.com \\ 5Professor do Curso de Agronomia, Universidade do Oeste de Santa Catarina-UNOESC. - São José do Cedro - SC; Brasil. E- \\ mail: acericato@gmail.com \\ ${ }^{\circ}$ Professor do Curso de Agronomia, Universidade do Oeste de Santa Catarina-UNOESC. - São José do Cedro - SC; Brasil. E- \\ mail: claudia.klein@unoesc.edu.br.
}

Artigo enviado em 04/07/2017, aceito em 01/10/2017 e publicado em 20/12/2017.

Resumo: A utilização de coinoculante é uma prática de baixo custo para culturas fixadoras de nitrogênio. O presente trabalho teve por objetivo avaliar o efeito da coinoculação na cultura da soja, em sistema de semeadura direta, no município de Guaraciaba - SC. O delineamento experimental foi conduzido em blocos casualizados, constituídos da combinação de inoculante padrão e Azospirillum, sendo: Não inoculado; Bradyrbizobium 100mL $50 \mathrm{~kg}^{-1}$ de sementes; Azospirillum 100mL 50kg-1 de sementes; Azospirillum 50mL + Bradyrbizobium 50mL 50kg-1 de sementes; Azospirillum $100 \mathrm{~mL}$ + Bradyrbizobium 100mL 50kg-1 de sementes; Azospirillum 150mL + Bradyrbizobium 150mL 50kg-1 de sementes; Azospirillum $200 \mathrm{~mL}+$ Bradyrbizobium 200mL 50 $\mathrm{kg}^{-1}$ de sementes. Foram avaliados rendimento de grãos, massa de mil sementes, índice SPAD (Soil Plant Analysis Development), teor de nitrogênio na folha e a eficiência econômica. O rendimento de grãos e massa de mil grãos foram submetidos à análise de variância pelo teste $\mathrm{F}$ e as diferenças entre médias foram comparadas pelo teste de Tukey $(\mathrm{P} \leq 0,05)$. A cultura da soja submetida à coinoculação obteve maior rendimento na dosagem de 400 $\mathrm{mL}$, chegando a elevar em até $13,3 \%$ em relação a não inoculada, tendo assim diferença estatística. Não houve diferença significativa para a variável massa de mil grãos. A eficiência econômica, apresentou margem líquida em todas as doses de inoculantes testadas, sendo que a mais expressiva foi também na maior dosagem de coinoculante de $400 \mathrm{~mL}$. A análise de regressão não revelou correlação entre as doses de coinoculante e os teores de N e o índice de SPAD.

Palavras-chave: Clorofilômetro. Eficiência econômica. Rendimento. Teor de nitrogênio.

Abstract - The use of coinoculant is a low cost practice for nitrogen fixing crops. The objective of this work was to evaluate the effect of co - inoculation on soybean crop, under no - tillage system, in the municipality of Guaraciaba - SC. The experimental design was conducted in randomized blocks, consisting of the combination of standard inoculant and Azospirillum, being: Not inoculated; Bradyrbizobium 100mL 50kg-1 of seeds; Azospirillum 100mL 50kg-1 of seeds; Azospirillum $50 \mathrm{~mL}+$ Bradyrhizobium 50mL 50kg-1 of seeds; Azospirillum $100 \mathrm{~mL}+$ Bradyrbizobium $100 \mathrm{~mL}$ 50kg-1 of seeds; Azospirillum $150 \mathrm{~mL}+$ Bradyrbizobium $150 \mathrm{~mL} 50 \mathrm{~kg}^{-1}$ of seeds; Arospirillum $200 \mathrm{~mL}+$ Bradyrbizobium $200 \mathrm{~mL} 50 \mathrm{~kg}^{-1}$ of seeds. Were evaluated, Grain yield, one thousand seed mass, SPAD index (Soil Plant Analysis Development), nitrogen content in leaf and economic efficiency were evaluated. The grain yield and the mass of one thousand grains were submitted to analysis of variance by the $\mathrm{F}$ test and the differences between averages were compared by the Tukey test $(\mathrm{P} \leq 0.05)$. The soybean crop submitted to co-inoculation obtained a higher yield in the $400 \mathrm{~mL}$ dosage, reaching up to $13.3 \%$ in relation to the uninoculated, thus having a statistical difference. There was no significant difference for the variable mass of a thousand grains. The economic efficiency, presented net margin in all doses of inoculants tested, and the most expressive was also in the higher co-inoculant dosage of $400 \mathrm{~mL}$. Regression analysis revealed no correlation between co-inoculant doses and $\mathrm{N}$ levels and SPAD index.

Keywords - Chlorophyllometer. Economic efficiency. Nitrogen content. 


\section{INTRODUÇÃO}

A fixação biológica de nitrogênio (FBN) pode ser feita através das bactérias do gênero Bradyrhizobium, a qual pode influir na diminuição dos custos de produção na cultura. Isso possibilita uma redução de adubos formulados contendo nitrogênio $(\mathrm{N})$, podendo obter maiores rendimentos de grãos (ZILLI et al., 2010).

A tecnologia de inoculação e reinoculação na cultura da soja tem proporcionado ganhos estatísticos significativos a 5\%, em relação ao controle sem reinoculação, os ganhos médios do uso da reinoculação a anual com Bradyrbizobium foram de 8,4\% e Reinoculação anual + Azospirillum no sulco foram de 16,1\% (HUNGRIA; NOGUEIRA, 2014).

A prática de coinoculação é uma técnica que consiste em fazer a combinação de duas bactérias, uma do gênero Bradyrbizobium e outra Azospirillum, essa prática pode promover um incremento no rendimento, mas essa tecnologia ainda é pouca conhecida pelos produtores, isso devido à falta de informação e trabalhos voltados a essa tecnologia.

Dentre várias dificuldades encontradas no manejo da soja, destacam-se a falta da prática de inoculação e coinoculação, mesmo que possa aumentar o rendimento da cultura. O que dificulta a adoção da tecnologia é a falta de informações em relação ao assunto e também que muitos produtores ainda não sabem se há viabilidade nessas práticas (HUNDRIA; NOGUEIRA; ARAÚJO, 2013).

Além disso com o passar dos anos tem se observado uma baixa eficiência agronômica na utilização do N. Isso acontece nas diversas culturas devido à adubação ser baseada principalmente em análises simples, como a visual, e nas recomendações tradicionais do manejo da cultura ou ainda observando as culturas antecessoras a ela (CARVALHO; SILVEIRA; SANTOS, 2012).

Dentre os métodos usados para verificar a necessidade de adubação, um deles é a avaliação do teor de clorofila nas folhas. Esse era mais demorado e exigia maior cuidado, pois havia o problema de coletar as amostras, as quais se tinha a necessidade de destruí-las, para só assim fazer o processo de extração e quantificação. Porém, recentemente desenvolveu-se um medidor portátil de clorofila (clorofilômetro), que permite fazer medições instantâneas do valor correspondente ao seu teor na folha sem destruí-la, essa alternativa estima o teor relativo desse pigmento na folha. $\mathrm{O}$ aparelho é utilizado para prever a necessidade de adubação nitrogenada em diversas culturas, pois a quantidade deste pigmento se compara ao teor de nitrogênio (ARGENTA; SILVA; BORTOLINI, 2001).

Desta forma, o objetivo desse trabalho é avaliar a eficiência técnica, agronômica e correlações das doses de coinoculante aplicadas em relação ao teor de $\mathrm{N}$ e o teor relativo de clorofila (Soil Plant Analysis Development SPAD) na folha, no estádio V5 e R2, utilizando as bactérias Azospirillum brasilense e Bradyrbizobium japonicum, na cultura da soja.

\section{MATERIAL E MÉTODOS}

O experimento foi conduzido no município de Guaraciaba, Santa Catarina, Brasil, situado nas coordenadas geográficas de $26^{\circ} 30^{\prime} 14^{\prime \prime}$ S e $53^{\circ} 31$ '22" W a 536 metros de altitude. O estudo foi conduzido entre os meses de outubro de 2014 a fevereiro de 2015.

Essa área foi escolhida por apresentar histórico da utilização em três anos consecutivos da cultura do milho (Zea mays L.), com sucessão de aveia (Avena sativa L.), sem a utilização de inoculante.

O clima da região, segundo classificação de Köppen, é do tipo Cfa. O solo da área experimental é classificado como LATOSSOLO VERMELHO Distrófico (EMBRAPA, 2013).

A análise de solo foi encaminhada para o Centro de Pesquisa para Agricultura Familiar (Epagri/Cepaf). A área revelou os seguintes resultados: Argila 46\%; pH (água) 5,5; Índice SMP 6,3; 7,6 $\mathrm{mg} \mathrm{dm}^{-3}$ de fósforo; $220,8 \mathrm{mg} \mathrm{dm}^{-3} \mathrm{de}$ potássio; 3,3\% de matéria orgânica; $0,0 \mathrm{cmol}_{\mathrm{c}} \mathrm{dm}^{-3} \mathrm{de}$ Alumínio; 6,5 $\mathrm{cmol}_{\mathrm{c}} \mathrm{dm}^{-3}$ de Cálcio; $2,4 \mathrm{cmol}_{\mathrm{c}} \mathrm{dm}^{-3} \mathrm{de}$ magnésio; CTC_pH 7.0 de $12,66 \mathrm{cmol}_{\mathrm{c}} \mathrm{dm}^{-3}$.

A aplicação de fósforo $(\mathrm{P})$, potássio $(\mathrm{K})$, foram calculadas segundo a Comissão de Química e Fertilidade do Solo - Rio Grande do Sul e Santa Catarina SBCS/CQFS (2004), sendo iguais para todas as parcelas. Com os resultados da análise química foi realizada a correção necessária de adubação para estimativa de produção de $3600 \mathrm{~kg} \mathrm{ha}^{-1}$

$\mathrm{O}$ estudo foi conduzido com delineamento em blocos casualizados, arranjados em faixas, com sete tratamentos. Cada tratamento teve quatro repetições totalizando 28 unidades experimentais. Cada parcela teve uma área de 4,8 $\mathrm{m}^{2}(2,4 \mathrm{~m}$ de largura $\mathrm{x} 2 \mathrm{~m}$ de comprimento) totalizando $19,2 \mathrm{~m}^{2}$ cada tratamento. A área total do experimento foi de $134,4 \mathrm{~m}^{2}$, sendo a área útil por parcela de $1,2 \mathrm{~m}^{2}(1,2 \mathrm{~m} \times 1,0 \mathrm{~m})$.

Os tratamentos foram constituídos por Azospirillum brasilense e Bradyrbizobium japonicum, coinoculados nas sementes de soja, sob diferentes dosagens, sendo: Não inoculado (Não Ino.); Bradyrbizobium $100 \mathrm{~mL} 50 \mathrm{~kg}^{-1}$ de sementes (Brad); Arospirillum $100 \mathrm{~mL} 50 \mathrm{~kg}^{-1}$ de sementes (Azo.); Azospirillum $50 \mathrm{~mL}+$ Bradyrbizobium 50mL 50kg-1 de sementes (Azo. $50 \mathrm{~mL}+$ Bra. $50 \mathrm{~mL}$ ); Azospirillum $100 \mathrm{~mL}+$ Bradyrbizobium 100mL 50 $\mathrm{kg}^{-1}$ de sementes (Azo. $100 \mathrm{~mL}+$ Brad. $100 \mathrm{~mL}) ;$ Arospirillum 150mL + 
Bradyrbizobium 150mL 50kg-1 de sementes (Azo. 150mL + Brad. $150 \mathrm{~mL}$ ); Azospirillum 200mL + Bradyrbizobium $200 \mathrm{~mL} 50 \mathrm{~kg}^{-1}$ de sementes (Azo. 200mL + Brad. 200 $\mathrm{mL})$.

Os inoculantes utilizados contém a bactéria Azospirillum brasilense, estirpes AbV5 e AbV6 e Bradyrbizobium japonicum, SEMIA 5079 e SEMIA 5080. A inoculação foi realizada com produto líquido, sendo utilizadas diferentes dosagens em $50 \mathrm{~kg}^{-1}$ semente. Antes de semear a soja foi realizado o manejo de dessecação da aveia, trinta dias antes da semeadura, com a cultivar AS 3570 IPRO (tecnologia INTACTA RR2 PRO $^{\circledR}$ ) (AGROESTE, 2014). A semeadura, foi realizada manualmente com espaçamento de $0,40 \mathrm{~m}$ entrelinhas e 7 $\mathrm{cm}$ entre plantas (13 plantas por metro linear).

Para determinação do rendimento, realizou-se a colheita de forma manual e posteriormente foi avaliado o componente de rendimento massa de mil grãos determinado com balança de precisão de legitimidade 0,001 gramas.

As amostras de tecido vegetal e SPAD foram coletados nos estádios V5 e R2. Através da metodologia proposta por Tedesco et al. (1995) foram determinados os teores de nitrogênio no tecido vegetal.

Os valores de SPAD foram determinados utilizando o clorofilômetro, realizando a leitura em 5 plantas dentro do 1,2 $\mathrm{m}^{2}$ útil, de cada parcela, as quais foram identificadas, para ser realizadas sempre nas mesmas. A avaliação das amostras se realizou em duas etapas, a primeira realizada no estádio V5, pois a partir desse estádio se tem uma maior atividade por parte dos nódulos, assim pode se obter uma leitura mais precisa. O próximo estádio avaliado foi em R2, por que neste estádio há elevada eficiência da fixação biológica (FAGAN et al., 2007).

Para se obter a margem líquida, foi feito a diferença de sacas do rendimento dos diferentes tratamentos em comparação ao tratamento não inoculado, utilizado um preço médio de $\mathrm{R} \$ 60,00 \mathrm{saca}^{-1}$, descontando o custo de mão de obra e dos inoculantes.

Para o rendimento da soja e a massa de mil grãos, foi realizado análise de variância com teste de significância Tukey, considerando a probabilidade de erro $(\mathrm{P} \leq 0,05)$. As análises foram realizadas com o programa estatístico ASSISTAT 7.7 beta (SILVA; AZEVEDO, 2009). O teor de $\mathrm{N}$ e SPAD foram tabulados e submetidos à análise de regressão, a qual não permitiu identificar as interferências entre as doses sobre as variáveis testadas.

\section{RESULTADOS E DISCUSSÕES}

Durante o período de realização do experimento a precipitações total registrada foi de $942 \mathrm{~mm}$, não ocorrendo déficit hídrico e a temperatura média registrada foi de $29,1{ }^{\circ} \mathrm{C}$ (Figura 1). Para que ocorra pleno crescimento e desenvolvimento na cultura da soja, o ideal é uma temperatura média de $20^{\circ} \mathrm{C}$ à $30^{\circ} \mathrm{C}$ (SYNGENTA, 2015) e a necessidade de hídrica entre 450 a $800 \mathrm{~mm}$ durante todo seu ciclo, o que podem variar dependendo das condições climáticas, do manejo da cultura e da duração de seu ciclo (EMBRAPA, 2011). Assim, tanto os totais de precipitação como os de temperatura média ficaram dentro dos parâmetros ideais para um bom desenvolvimento de seu ciclo, interferindo diretamente para os altos rendimentos em todos os tratamentos testados.

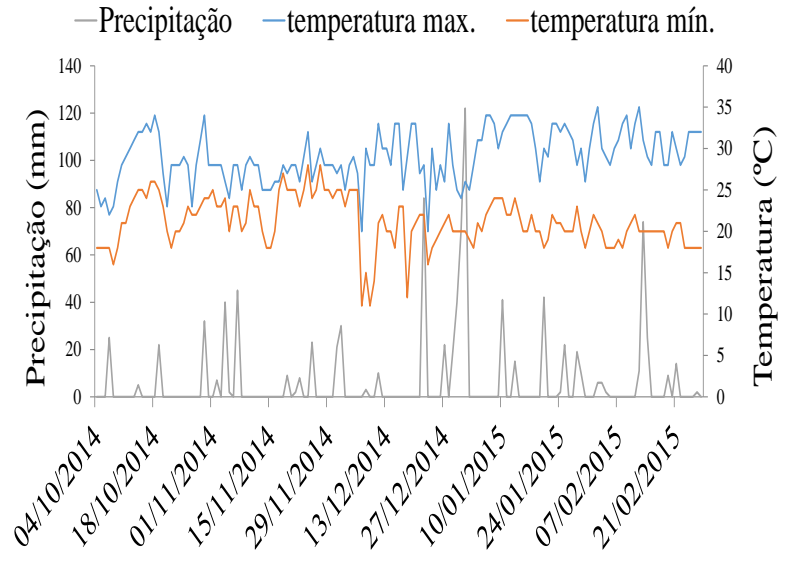

Figura 1. Dados meteorológicos da precipitação e temperatura.

$\mathrm{Na}$ variável rendimentos de grãos (Figura 2), observou-se diferença significativa, onde os dados obtidos no experimento em relação a diferentes tratamentos, apresentaram resultados que coincidem com os resultados de outros trabalhos (HUNGRIA; CAMPO; MENDES, 2007).

O alto rendimento em todos os tratamentos se deve as condições climáticas favoráveis, ocorridas no período do estabelecimento da cultura, sendo que proporcionou um bom desenvolvimento na soja.

Avaliando os dados apresentados para rendimento, percebe-se que houve diferença estatística na dosagem de $400 \mathrm{~mL}$ (Azo. 200mL + Brad. $200 \mathrm{~mL}$ ), com rendimento de $6.258 \mathrm{~kg} \mathrm{ha}^{-1}$, obtendo um aumento de $832 \mathrm{~kg}$ ou $13,3 \% \mathrm{em}$ comparação ao tratamento não inoculado, que foi o menor com $5.426 \mathrm{~kg} \mathrm{ha}^{-1}$. Este elevado rendimento 
de $6.258 \mathrm{~kg} \mathrm{ha}^{-1}$ equivale a 104,3 sacas ha-1 de $60 \mathrm{~kg} \mathrm{ha}^{-1}$, porém deve-se considerar que não teve perdas de colheita.

No tratamento somente com bactérias de Azospirillum, teve um rendimento de $5.941 \mathrm{~kg} \mathrm{ha}^{-1}$, que ficou acima do tratamento somente com Bradyrbizobium, o qual, rendeu $5.603 \mathrm{~kg} \mathrm{ha}^{-1}$, proporcionando um aumento de $338 \mathrm{~kg}$ ou 5,7\%, isso provavelmente ocorreu devido a capacidade dessas bactérias promoverem um maior crescimento radicular, o que possibilita a extração de mais nutrientes num volume maior de solo (BÁRBARO et al., 2008).

Com o sistema radicular maior, pode-se ter aumento da quantidade de nódulos, os quais podem realizar a absorção de $\mathrm{N}$ e junto com as bactérias diazotróficas que aumentam o tamanho das raízes e o volume delas, elas também podem fornecer diversos efeitos benéficos da interação com os fungos micorrízicos arbusculares, como o incremento da absorção de fósforo (P). Isso se torna importante, pois pode-se aumentar o rendimento, diminuindo a utilização de insumos químicos como os fosfatados e também os nitrogenados (DE SOUZA et al., 2011).

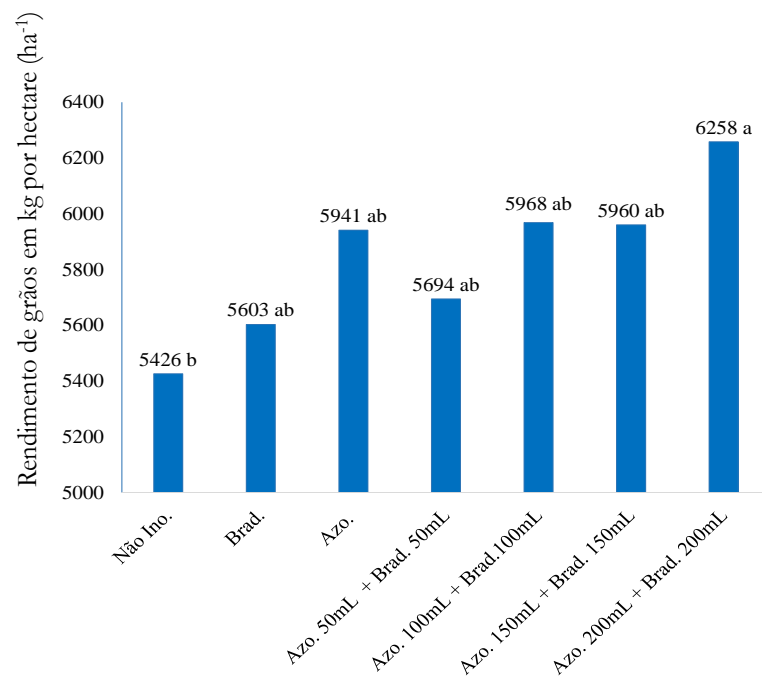

Figura 2. Rendimento de grãos em $\mathrm{kg}$ por hectare $\left(\mathrm{ha}^{-1}\right)$. Não inoculado (Não Ino); Bradythizobium $100 \mathrm{~mL}^{50 \mathrm{~kg}^{-1}}$ de sementes (Brad); Azospirillum $100 \mathrm{~mL} 50 \mathrm{~kg}^{-1}$ de sementes (Azo.); Azospirillum 50mL + Bradyrhizobium $50 \mathrm{~mL} 50 \mathrm{~kg}^{-1}$ de sementes; (Azo. $50 \mathrm{~mL}+$ Bra. $50 \mathrm{~mL}$ ); Azospirillum $100 \mathrm{~mL}+$ Bradyrhizobium $100 \mathrm{~mL} 50 \mathrm{~kg}^{-1}$ de sementes (Azo. 100mL + Brad. $100 \mathrm{~mL}$ ); Azospirillum $150 \mathrm{~mL}+$ Bradyrhizobium 150mL 50kg-1 de sementes (Azo. $150 \mathrm{~mL}+$ Brad. $150 \mathrm{~mL}) ;$ Azospirillum $200 \mathrm{~mL}+$

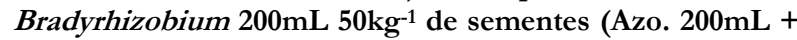
Brad. $200 \mathrm{~mL}$ ). Médias seguidas por letras diferentes em minúsculo diferem pelo teste estatístico Tuckey $(P \leq 0,05)$. Coeficiente de Variação (CV) de 5,87\%.
Além do aumento do sistema radicular e da nodulação, o rendimento da cultura também está relacionado aos bons fatores climáticos, que ocorreram durante o ciclo, devido a importância da demanda hídrica para seu desenvolvimento, desde o estado vegetativo, período de florescimento e maturação fisiológica.

$\mathrm{Na}$ variável massa de mil grãos (Figura 3), não se observou diferença significativa nos dados obtidos no estudo, em relação aos diferentes tratamentos, porém houve um aumento na massa, se compararmos os tratamentos do Bradyrbizobium e Azo. $50 \mathrm{~mL}+$ Brad. $50 \mathrm{~mL}$, com o tratamento de Azo. 200mL + Brad. 200mL. Este efeito pode estar relacionado a uma maior floração e assim, um maior número de grãos por planta, diminuindo a destinação de fotoassimilados para cada grão.

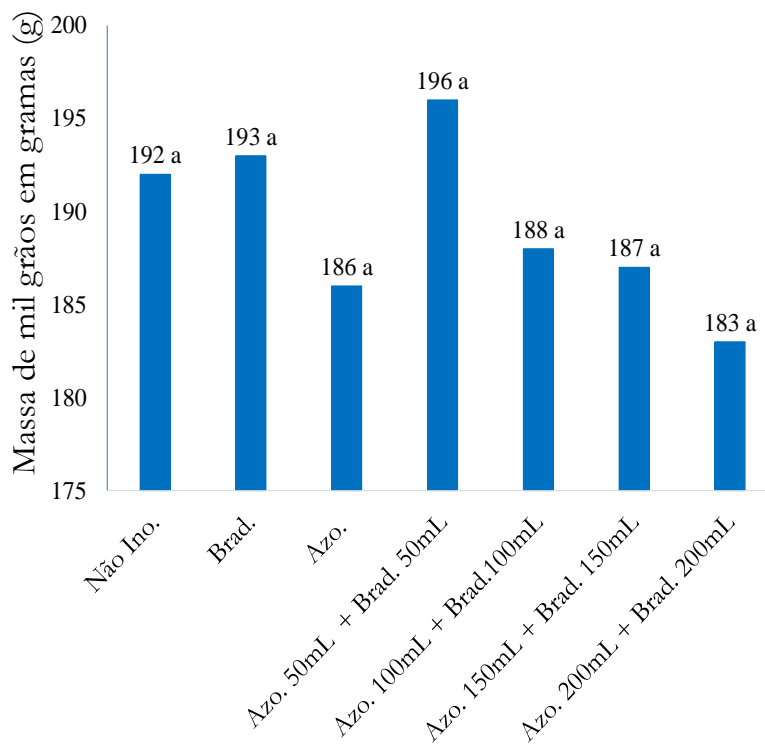

Figura 3. Massa de mil grãos em gramas (g).

Não inoculado (Não Ino); Bradyrhizobium $100 \mathrm{~mL} 50 \mathrm{~kg}^{-1}$ de sementes (Brad); Azospirillum $100 \mathrm{~mL} 50 \mathrm{~kg}^{-1}$ de sementes (Azo.); Azospirillum 50mL + Bradyrhizobium $50 \mathrm{~mL} 50 \mathrm{~kg}^{-1}$ de sementes; (Azo. 50mL + Bra. 50mL); Azospirillum $100 \mathrm{~mL}+$ Bradyrhizobium $100 \mathrm{~mL} 50 \mathrm{~kg}^{-1}$ de sementes (Azo. $100 \mathrm{~mL}+$ Brad. $100 \mathrm{~mL}$ ); Azospirillum $150 \mathrm{~mL}+$ Bradyrhizobium $150 \mathrm{~mL} 50 \mathrm{~kg}^{-1}$ de sementes (Azo. $150 \mathrm{~mL}+$ Brad. $150 \mathrm{~mL}) ;$ Azospirillum $200 \mathrm{~mL}+$ Bradyrhizobium 200mL 50 kg-1 de sementes (Azo. 200mL + Brad. $200 \mathrm{~mL}$ ). Médias seguidas pela mesma letra em minúsculo não diferem pelo teste estatístico Tuckey $(\mathbf{P} \leq$ 0,05). Coeficiente de Variação (CV) de 4,71\%.

Conforme Floss (2011), o enchimento de grãos está diretamente relacionado com a atividade fotossintética e fotorespiratória da planta. Os produtos da fotossíntese são transportados pelo floema, levando fotoassimilados como 
açucares e sacarose para as partes de crescimento e aos grãos.

Esse movimento dos fotoassimilados pode ser chamada de relação fonte-dreno. As fontes são as regiões da planta, onde ocorre a síntese, como por exemplo, as folhas que estão realizando fotossíntese e os drenos são as regiões de armazenamento ou crescimento, como ápices meristemáticos, raízes e inclusive os grãos. Quando a planta está num estádio vegetativo a translocação ocorre geralmente para os ápices caulinares e para as raízes. Já na fase reprodutiva o transporte desses açucares se direciona para os grãos (FLOSS, 2011).

Desta forma as plantas que possuíam menor número de grãos, tiveram um maior direcionamento de fotoassimilados, fazendo com que a estrutura dessas plantas, fornecem maior reserva de energia e consequentemente esses tratamentos possuíram massa de grãos superior aos demais, mesmo com menor rendimento, quando comparado ao tratamento de Azo. $200 \mathrm{~mL}+$ Brad. $200 \mathrm{~mL}$, que teve o maior rendimento com maior quantidade de grãos por planta, porém com menor massa por grão.

$\mathrm{Na}$ eficiência econômica observou-se presença de margem líquida em todos os tratamentos, quando comparados com o não inoculado (Tabela 1).

A maior diferença de rendimento foi no tratamento de Brad. $200 \mathrm{~mL}+$ Azo. 200mL comparativamente ao Não Ino., em valores de $832 \mathrm{~kg} \mathrm{ha}^{-1}$ ou 13,9 sacas ha-1. Essa quantidade calculada a um preço médio de $\mathrm{R} \$ 60,00$ sacas $^{-1}$, se equivale a $\mathrm{R} \$ 832,00 \mathrm{ha}^{-1}$, sendo que esse tratamento teve um custo de $\mathrm{R} \$ 45,16 \mathrm{ha}^{-1}$ obteve-se assim, uma margem líquida de $\mathrm{R} \$ 786,84 \mathrm{ha}^{-1}$, tornando a prática de coinoculação economicamente viável.

Tabela 1. Custo e margem líquida da inoculação e coinoculação por hectare (ha-1).

\begin{tabular}{|c|c|c|c|c|c|c|c|}
\hline Tratamentos & $\begin{array}{c}\text { Custo de } \\
\text { mão de } \\
\text { obra }(\mathrm{R} \$)\end{array}$ & $\begin{array}{l}\text { Custo } \\
\mathrm{R} \$ \mathrm{~kg}^{-1}\end{array}$ & $\begin{array}{c}\text { Custo } \\
\left(\mathrm{R} \$ \mathrm{ha}^{-1}\right) \\
(62 \mathrm{Kg})\end{array}$ & $\begin{array}{c}\text { Total } \\
\text { Custos } \\
\left(\mathrm{R} \$ \mathrm{ha}^{-1}\right)\end{array}$ & $\begin{array}{l}\text { Diferença } \\
\text { de sacas }\end{array}$ & $\begin{array}{c}\text { Preço } \\
\left(\mathrm{R} \$ \mathrm{saca}^{-1}\right)\end{array}$ & $\begin{array}{l}\text { Margem Líquida } \\
\qquad\left(\mathrm{R} \$ \mathrm{ha}^{-1}\right)\end{array}$ \\
\hline Não Ino. & 0,00 & 0,00 & 0,00 & 0,00 & 0,00 & 60,00 & 0,00 \\
\hline Brad. & 3,00 & 0,10 & 6,20 & 9,20 & 2,9 & 60,00 & 168,80 \\
\hline Aqo. & 3,00 & 0,24 & 14,88 & 17,88 & 8,6 & 60,00 & 498,12 \\
\hline $\begin{array}{l}\text { Azo. } 50 \mathrm{~mL}+\text { Brad. } 50 \mathrm{~mL} \\
\text { Azo. } 100 \mathrm{~mL}+\text { Brad. }\end{array}$ & 3,00 & 0,17 & 10,54 & 13,54 & 4,5 & 60,00 & 256,46 \\
\hline $\begin{array}{c}100 \mathrm{~mL} \\
\text { Azo. } 150 \mathrm{~mL}+\text { Brad. } 150\end{array}$ & 3,00 & 0,34 & 21,08 & 24,08 & 9,0 & 60,00 & 515,92 \\
\hline $\begin{array}{c}\mathrm{mL} \\
\text { Azo. } 200 \mathrm{~mL}+\text { Brad. }\end{array}$ & 3,00 & 0,51 & 31,62 & 34,62 & 8,9 & 60,00 & 499,38 \\
\hline $200 \mathrm{~mL}$ & 3,00 & 0,68 & 42,16 & 45,16 & 13,9 & 60,00 & 786,84 \\
\hline
\end{tabular}

A busca por qualificação juntamente com as informações técnicas melhorou a eficiência no rendimento da soja, tornando economicamente viável, a prática da coinoculação. Essa é uma técnica rentável para o cultivo da soja, uma vez que esse método apresentou vantagens de rendimento e ganhos econômicos, mostrando margem líquida positiva e que fornece viabilidade de utilização da coinoculação.

$\mathrm{Na}$ análise dos teores de $\mathrm{N}$ e no valor SPAD, teve variação entre os estádios fisiológicos da planta (Tabela 02).
O SPAD da soja no estádio V5 obteve média de 33,76 (variação de 33,44 a 34,66) e com os teores de $\mathrm{N}$ variando de 4,03\% (100 mL de coinoculação) a 5,33\% quando não inoculado, onde a faixa ideal para a cultura da soja é de $4,5 \%$ a $5,5 \%$, ou seja, é nessa faixa onde a planta tem este nutriente, em quantias ideais para completar seu ciclo (FLOSS, 2011).

Segundo Argenta et al. (2001), a utilização do clorofilômetro se torna importante, pois consegue avaliar o estado nutricional da planta, indicando o teor de clorofila, e assim, pode-se saber o teor de $\mathrm{N}$ que tem nas folhas em tempo real, porém não se sabe no 
momento a quantia de adubação nitrogenada, a ser aplicada, no entanto é um indicador das condições fisiológicas, que acaba sendo benéfico o uso desta tecnologia. Segundo Pereira (2016), o teor de clorofila na folha é utilizado para predizer o nível nutricional de nitrogênio (N) em plantas, devido ao fato de a quantidade desse pigmento correlacionar-se positivamente com teor de $\mathrm{N}$ na planta.

As clorofilas são pigmentos responsáveis pela captura de luz usada na fotossíntese, sendo elas essenciais na conversão da radiação luminosa em energia química, na forma de ATP e NADPH. Assim, as clorofilas estão relacionadas com a eficiência fotossintética das plantas e, consequentemente com seu crescimento e adaptabilidade aos diferentes ambientes. Tradicionalmente os métodos utilizados para determinação do teor de clorofila requerem destruição das folhas, o que é uma desvantagem em estudos que visem determinar o efeito da ontogenia da folha no grau de esverdeamento. Além disso, esses métodos são muito demorados e onerosos. $\mathrm{Na}$ década de 90 foi disponibilizado um equipamento capaz de gerar grandezas relacionadas com os teores de clorofila, o clorofilômetro Soil Plant Analysis Development (SPAD-502, Minolta, Japão). Esse instrumento é portátil e fornece leituras que podem se relacionar com o teor de clorofila presente na folha. Permite medições de forma rápida (poucos segundos) e prática, ainda em campo a um custo baixo. Devido à alta correlação que existe entre os teores de nitrogênio e clorofila (MARENCO; LOPES, 2007), um dos usos principais do SPAD-502 tem sido para estimar o teor de nitrogênio da folha, em culturas.

Tabela 2. Teor de nitrogênio e valor de SPAD nas diferentes doses de coinoculação e estádios vegetativos e reprodutivos da Soja.

\begin{tabular}{ccccc}
\hline & \multicolumn{2}{c}{ Teor de N $(\%)$} & \multicolumn{2}{c}{ SPAD } \\
\hline Tratamentos/Estádio da Soja & V5 & R2 & V5 & R2 \\
\hline Não Ino. & $5,33 \mathrm{a}$ & $5,33 \mathrm{a}$ & $33,44 \mathrm{a}$ & $29,09 \mathrm{a}$ \\
Brad. & $4,81 \mathrm{ab}$ & $4,63 \mathrm{a}$ & $34,53 \mathrm{a}$ & $30,21 \mathrm{a}$ \\
Azo. & $5,42 \mathrm{a}$ & $5,46 \mathrm{a}$ & $34,23 \mathrm{a}$ & $29,51 \mathrm{a}$ \\
Azo. 50mL + Brad. 50mL & $4,02 \mathrm{~b}$ & $4,85 \mathrm{a}$ & $33,47 \mathrm{a}$ & $29,48 \mathrm{a}$ \\
Azo. 100mL + Brad. 100mL & $5,25 \mathrm{a}$ & $4,33 \mathrm{a}$ & $33,73 \mathrm{a}$ & $30,86 \mathrm{a}$ \\
Azo. 150 mL + Brad. 150 mL & $5,11 \mathrm{a}$ & $4,41 \mathrm{a}$ & $34,66 \mathrm{a}$ & $32,09 \mathrm{a}$ \\
Azo. 200mL + Brad. 200mL & $4,85 \mathrm{ab}$ & $4,02 \mathrm{a}$ & $33,50 \mathrm{a}$ & 4,4
\end{tabular}

Não inoculado (Não Ino); Bradyrbizobium 100mL 50kg-1 de sementes (Brad); Azospirillum 100mL 50kg-1 de sementes (Azo.); Azospirillum 50mL + Bradyrbizobium 50mL 50kg-1 de sementes; (Azo. 50mL + Bra. 50mL); Azospirillum 100mL + Bradyrbizobium 100mL 50kg-1 de sementes (Azo. 100mL + Brad. $100 \mathrm{~mL}$ ); Azospirillum 150mL + Bradyrhizobium 150mL 50kg-1 de sementes (Azo. 150mL + Brad. $150 \mathrm{~mL}$ ); Azospirillum 200mL + Bradyrhizobium 200mL 50kg-1 de sementes (Azo. 200mL + Brad. $200 \mathrm{~mL}$ ).

No estádio R2 não houve influência das doses de coinoculação, em relação ao teor de $\mathrm{N}$ na folha e ao SPAD. No estádio R2 e nos tratamentos de Brad. 150 $\mathrm{mL}+$ Azo. $150 \mathrm{~mL}$ e no Brad. $200 \mathrm{~mL}+$ Azo. $200 \mathrm{~mL}$, foram respectivamente $4,42 \%$ a $4,03 \%$ de $\mathrm{N}$ na folha.

Para as leguminosas, como no caso da cultura da soja, o nitrogênio é exigido em grande quantidade em todas as fases de seu desenvolvimento, mas é na fase reprodutiva que há mais necessidade desse nutriente. De toda a quantia que a planta utiliza, grande parte é transportada para os grãos e o restante fica nas folhas, caule e raízes (HUNGRIA; CAMPO; MENDES, 2001).

Segundo Fagan et al. (2007), é em R2 onde ocorre um alto acúmulo de matéria seca, e nutrientes pela planta. Esse processo se inicia em folhas, hastes e raízes, começando a partir desse momento destinar parte dos nutrientes inclusive o $\mathrm{N}$, para as flores e depois para vagens e sementes, diminuindo assim o teor de $\mathrm{N}$ nas folhas.

Através do IRC (Índice Relativo de Clorofila), avaliado no clorofilômetro, pode-se saber o nível de $\mathrm{N}$ da folha, por esse fazer parte da clorofila, que é constituída por pigmentos verdes responsáveis pela captura de luz, usada na fotossíntese. O clorofilômetro vem sendo utilizado na quantificação de clorofila das folhas, proporcionando mais rapidez e simplicidade, mas principalmente por possibilitar uma avaliação não destrutiva da amostra e poder acompanhar as condições nutricionais da planta em tempo real (FONSECA et al., 2012).

Segundo Uddling et al. (2007), para se obter a máxima precisão do instrumento é recomendado que o SPAD-502 seja calibrado sempre que uma nova 
espécie esteja sendo estudada. A falta de linearidade entre os teores relativos (obtidos pelo clorofilômetro) e os teores absolutos de clorofila, observados na literatura, sugere que a distribuição da clorofila na superfície da folha apresenta certa desuniformidade, sobretudo em folhas bem esverdeadas o que pode levar a uma subestimação dos valores do SPAD em folhas com altos teores de clorofila. Até o presente momento não há trabalhos que avaliem o aumento de produtividade, com adubos nitrogenados e os teores na cultura da soja (SCHEFER et al., 2016).

\section{CONCLUSÕES}

A utilização da coinoculação, proporciona aumento no rendimento da soja, chegando a elevar em até $13,3 \%$, comparativamente a não inoculada, obtendo uma margem líquida de $\mathrm{R} \$ 786,00$, devido ao baixo custo da técnica de coinoculação.

A massa de mil grãos foi inversamente proporcional as doses de coinoculação testadas, pois essas induziram uma maior floração e assim, um maior número de grãos por planta, diminuindo a destinação de fotoassimilados para cada grão.

A evolução do índice SPAD foi proporcional a dose de coinoculação realizada, principalmente no estádio R2.

Os teores de $\mathrm{N}$ no estádio $\mathrm{R} 2$ foram inversamente proporcionais as doses de coinoculante, atribuindo a maior rendimento e consequentemente a maior relação fonte dreno.

\section{REFERÊNCIAS}

AGROESTE. Descrição da cultivar AS 3570 IPRO. $2014 . \quad$ Disponível em: http://www.agroeste.com.br/intactarr2pro/variedades _mostra.php?tipo=as_3570. Acesso em 09 mai. 2015.

ARGENTA, G. DA SILVA, P. R. F. BORTOLINI, C. G. FORSTHOFER, E. L. STRIEDER, M. L. Relação da leitura do clorofilômetro com os teores de clorofila extraível e de nitrogênio na folha de milho. Revista Brasileira de Fisiologia Vegetal. Porto Alegre/RS, v. 13 n. 2, 2001.
ARGENTA, G.; SILVA, P. R. F. D; BORTOLINI, C. G. Clorofila na folha como indicador do nível de nitrogênio em cereais. Ciência Rural. Rio Grande do Sul. 2001. v. 31. n. 4. Disponível em: http://www.scielo.br/scielo.php?pid=S0103-

$84782001000400027 \&$ script $=$ sci_arttext. Acesso em 23 out. 2014 .

BÁRBARO, I. M. BRANCALIÃO, S. R. TICELLI, M. MIGUEL, F. B. DA SILVA, J. A. A. Técnica alternativa: co-inoculação de soja com Azospirillum e Bradyrbizobium visando incremento de produtividade. Informações Tecnológicas. Infobibos. Capinas, São Paulo. 2008. Disponível em: http://www.infobibos.com/Artigos/2008_4/coinocula cao/index.htm. Acesso em: 15 set. 2014.

CARVALHO, M. A. D. F.; SILVEIRA, P. M. D.; SANTOS, A. B. D. Utilização do clorifilômetro para racionalização da adubação nitrogenada nas culturas do Arroz e do Feijoeiro. Goiás. Embrapa. 2012. 14p. Disponível Em: http://www.cnpaf.embrapa.br/transferencia/informa coestecnicas/publicacoesonline/comunicadotecnico_2 05.pdf. Acesso em 23 out. 2014.

DE SOUZA, F. A. GOMES, E. A. VASCONCELOS, M. J. V. DE SOUSA, S. M. Micorrizas Arbusculares: Perspectivas para aumento da eficiência de aquisição de Fósforo (P) em Poaceae Gramíneas. ed. 1º. Embrapa Milho e Sorgo, 2011. Documentos/Embrapa Milho e Sorgo 134. 30 p.

EMBRAPA 2011. Sistemas de produção, tecnologias de produção de soja Região Central do Brasil. 2011. p. 11-12, Out. 2011.

EMBRAPA 2013. Centro nacional de pesquisa de solos. Sistema brasileiro de classificação do solo. ed $3^{\circ}$, Brasília, DF. 2013. 353p.

FAGAN, E. B.; MEDEIROS, S. L. P.; MANFRON, P. A.; CASAROLI, D.; SIMON, J.; DOURADO NETO, D.; LIER, Q. D. J. V.; SANTOS, O. S.; MÜLLER, L. FISIOLOGIA DA FIXAÇÃO BIOLÓGICA DO NITROGÊNIO EM SOJA REVISÃO. Revista da FZVA. Uruguaiana, v.14, n.1, p. 89-106. 2007.

FLOSS, E. L. Fisiologia das plantas cultivadas: nitrogênio. Universidade de Passo Fundo, Passo Fundo, RS, 5. ed, 2011. 
FONSECA, P. R. B. DA. FERNANDES, M. G. DUTRA, F. DE SOUZA, T. A. PONTIM, B. C. Á. Uso do SPAD-502 na avaliação dos teores foliares de clorofila, e, híbridos de milho, (Zea mays L.) BT e isogênico. Revista Verde (Mossoró - RN - Brasil). 2012. v.7, n.1, p. 56 - 60. Disponível em: http://www.gvaa.com.br/revista/index.php/RVADS /article/view/1087/pdf_359. Acesso em 14 mai. 2015.

HUNDRIA, M.; NOGUEIRA, M.A.; ARAUJO, R.S. Tecnologia de coinoculação da soja com Bradyrbiz̧obium e Azospirillum: incrementos no rendimento com sustentabilidade e baixo custo. In: Reunião de Pesquisa de Soja da Região Central do Brasil, 33, 2013, Londrina.

HUNGRIA, M. CAMPO, R. J. MENDES, I. C. A importância do processo de fixação biológica do nitrogênio para a cultura da soja: componente essencial para a competitividade do produto brasileiro. Londrina, Brasil, Londrina: Embrapa Soja: Embrapa Cerrados, 2007. 80p. Documentos/Embrapa Soja, n. $80 \mathrm{p}$.

HUNGRIA, M.; CAMPO, R. J.; MENDES, I. C. Fixação biológica do nitrogênio na cultura da soja. EMBRAPA, 2001, 48 p. (Circular Técnica, 35). Disponível em: http://www.infoteca.cnptia.embrapa.br/bitstream/do c/459673/1/circTec35.pdf. Acesso em 22 de out. 2014.

HUNGRIA, M.; NOGUEIRA, M. A. Tecnologia De Coinoculação Rizóbios e Aqospirillum em Soja e Feijoeiro. Embrapa Soja. Folder, 02/2014.

MARENCO, R.A.; LOPES, N.F. Fisiologia vegetal: fotossintese, respiraşão, relações hídricas e nutrição mineral. 2 ed. Editora UFV, Viçosa, MG. 469 p. 2007

PEREIRA, M. T. de T. Clorofilometria por imagem digital aplicada à cultura do milho (Zea mays L.). 2016. 68 f. Dissertação (Mestrado) - Curso de Programa de Pósgraduação em Tecnologia e Gestão da Inovação, Universidade Comunitária da Região de Chapecó (Unochapecó), Chapecó, 2016.

SCHEFER, A. CIPRIANI, K..; CERICATO, ALCEU.; SORDI ANDRÉ.; LAJÚS, C. R. Eficiência Técnica e Econômica da Cultura da Soja Submetida À Aplicação de Fertilizantes Nitrogenados em Semeadura e Coberturas. Scientia agrária. v. 17 n. 2 Curitiba abril/maio. 2016 p. $14-20$
SILVA, F. A. S.; AZEVEDO, C. A. V. Principal components analysis in the software assistat-statistical attendance. In: World Congress On Computers In: Agriculture, v. 7, Reno-NV-USA: American. Society of Agricultural and Biological Engineers, 2009.

SOCIEDADE BRASILEIRA DE CIÊNCIA DO SOLO/COMISSÃO DE QUÍMICA E FERTILIDADE DO SOLO - SBCS/CQFS RS/SC. Manual de adubação e calagem para os Estados do Rio Grande do Sul e de Santa Catarina. Sociedade Brasileira de Ciência do Solo. 10. ed. Porto Alegre, SBCS - Núcleo Regional Sul/UFRGS, 2004. 400p.

SYNGENTA. Guia de sementes de soja para o Sul. Morfologia e Fisiologia da planta. 2015.

TEDESCO, M. J. GIANELLO, C. BISSANI, C. A. BOHNEN, H. VOLKWEISS, S. J. Análise de solo, Plantas e Outros Materiais. Porto alegre, 1995. p.93.

UDDLING, J.; GELANQ, A. J.; PIIKKI, K.; PLEIJEL, H. Evaluating the relationship between leaf chlorophyll concentration and SPAD-502 chlorophyll meter readings. Photosynth. Res., 91: 37-46, 2007.

ZILLI, J. É.; GIANLUPPI, V.; CAMPO, R. J.; ROUWS, J. R. C.; HUNGRIA, M. Inoculação da soja com Bradyrbizobium no sulco de semeadura alternativamente á inoculação de sementes. Revista Brasileira de Ciência do solo. Viçosa. v. 34. 7p. 2010. Disponível em: http://www.scielo.br/pdf/rbcs/v34n6/11.pdf. Acesso em: 22 out. 2014. 